\title{
QUELLE RÉVOLUTION POUR LES BINATIONAUX ?
}

Le rôle des Franco-Tunisiens dans la chute de la dictature et dans la transition politique

Vincent Geisser

Centre d'information et d'études sur les migrations internationales | « Migrations Société »

2012/5 N 143 | pages 155 à 178

ISSN 0995-7367

Article disponible en ligne à l'adresse :

https://www.cairn.info/revue-migrations-societe-2012-5-page-155.htm

Distribution électronique Cairn.info pour Centre d'information et d'études sur les migrations internationales.

(C) Centre d'information et d'études sur les migrations internationales. Tous droits réservés pour tous pays.

La reproduction ou représentation de cet article, notamment par photocopie, n'est autorisée que dans les limites des conditions générales d'utilisation du site ou, le cas échéant, des conditions générales de la licence souscrite par votre établissement. Toute autre reproduction ou représentation, en tout ou partie, sous quelque forme et de quelque manière que ce soit, est interdite sauf accord préalable et écrit de l'éditeur, en dehors des cas prévus par la législation en vigueur en France. Il est précisé que son stockage dans une base de données est également interdit. 


\title{
QUELLE RÉVOLUTION POUR LES BINATIONAUX ?
}

\section{Le rôle des Franco-Tunisiens dans la chute de la dictature et dans la transition politique}

Vincent GEISSER *

\begin{abstract}
«Nous sommes la seconde génération. Il y a une diaspora tunisienne qui est importante. Notre objectif est de mobiliser cette diaspora pour que plus jamais le gouvernement français ne soutienne en Tunisie une dictature comme celle de Ben Ali. Nous voulons nous organiser pour qu'il y ait au sein de la diaspora une conscience citoyenne, afin que les choses ne se reproduisent plus comme dans le passé ».

Wajdi Limam, président-fondateur de l'association Union pour la Tunisie (UNIT).
\end{abstract}

En France, le 15 janvier 2011 , moins de 24 heures après la fuite du président Ben Ali en Arabie Saoudite, plusieurs milliers de manifestants convergent vers les lieux symboliques, place de la République à Paris et La Canebière à Marseille, afin de communier dans un esprit patriotique et scander en cœur le slogan : "Nous n'aurons plus jamais peur ! " ${ }^{1}$. La situation est inédite dans l'Hexagone car, contrairement aux décennies précédentes, où les mobilisations contre le régime autoritaire parvenaient à rassembler à peine quelques dizaines de personnes, pour la plupart issues des rangs de l'extrême gauche, des associations des droits de l'homme ou des milieux islamistes ${ }^{2}$, il s'agissait cette fois-ci de manifestations multitudinaires.

* Chercheur à l'Institut français du Proche-Orient (IFPO), Beyrouth, Liban ; président du CIEMI.

1. Observation participante conduite entre décembre 2010 et juin 2011 dans plusieurs villes françaises, notamment Paris et sa banlieue, Lyon, Aix-en-Provence et Marseille.

2. Cf. BÉCHIR-AYARI, Michaël, S'engager en régime autoritaire : gauchistes et islamistes dans la Tunisie indépendante, thèse de doctorat, Université Paul Cézanne, Aix-en-Provence, 2009, 476 p. BÉCHIR-AYARI, Michaël, "Rester le même tout en devenant un autre : les 'islamistes' tunisiens exilés en France", Maghreb-Machrek, n 194, hiver 2007-2008, dossier "Les mouvements islamistes des armes aux urnes", pp. 55-73. 


\section{La renaissance politique de la majorité silencieuse}

Pour la première fois, des Tunisiens anonymes sont descendus massivement dans la rue pour manifester leur soutien au changement politique et soutenir le processus de démocratisation. La mobilisation ultraminoritaire des "professionnels de la contestation" a laissé la place à un vaste rassemblement intergénérationnel et interclassiste, où les chibanis issus des premières vagues d'immigration tunisienne en France côtoient les épiciers djerbiens, les boulangers originaires des régions présahariennes (Ghomrassen, Tataouine, Douiret) ${ }^{3}$, les jeunes venus faire leurs études universitaires dans l'Hexagone ${ }^{4}$, les expatriés appartenant aux professions libérales et intellectuelles (médecins, avocats, hommes d'affaires, artistes...), noyés dans la masse de ce qu'on appelle communément les "binationaux", filles et fils de travailleurs migrants, majoritairement de nationalité française.

En apparence, rien ne pouvait laisser penser que ces Ould el França ${ }^{5}$, faiblement impliqués dans les enjeux sociopolitiques de la société d'origine de leurs parents, réduite généralement à sa dimension estivale et exotique (pays de vacances et de cocagne) ${ }^{6}$, puissent s'identifier avec une telle force aux mouvements protestataires du bled, au point de considérer la Révolution du 14 janvier comme la leur. Car, finalement, ce qu'a révélé en creux la "surprise" de cet engagement collectif des Français d'origine tunisienne, c'est l'incapacité des sciences sociales à conceptualiser non seulement les formes multiples d'identification des "héritiers de l'immigration" aux enjeux politiques des États d'origine, au-delà de la représentation binaire allégeance/intégration, mais aussi l'articulation complexe entre la problématique migratoire et celle des mobilisations sociales, qui fait figure aujourd'hui d'impensé et de parent pauvre de la sociologie des migrations, comme le déplore très justement Antoine Dumont : " Fréquemment analysées de manière distincte, mobilisation sociale et migration internationale peuvent être étroitement articulées dans les pratiques et les représentations des acteurs transnationaux 11 .

3. Cf. BOUBAKRI, Hassan, "Modes de gestion et réinvestissements chez les commerçants tunisiens à Paris", Revue Européenne des Migrations Internationales, vol. 1, n 1, septembre 1985, pp. 49-64.

4. Cf. GEISSER, Vincent (sous la direction de), Diplômés maghrébins d'ici et d'ailleurs : trajectoires sociales et itinéraires migratoires, Paris : CNRS Éditions, 2000, 305 p.

5. Formule dialectale tunisienne que l'on peut traduire approximativement par "natifs de France" ou "enfants de France", et qui est parfois utilisée péjorativement dans le pays d'origine pour mettre en doute la "tunisianité" des enfants d'émigrés.

6. Cf. ORIOL, Michel, "L'effet Antée ou les paradoxes de l'identité symbolique”, Peuples Méditerranéens, $\mathrm{n}^{\circ} 24$, juillet-septembre 1983, pp. 45-60.

7. DUMONT, Antoine, "De Redeyef à Nantes : mobilisation sociale et migration internationale", Revue Tiers Monde, hors série, 2011, pp. 47-66. 
Toutefois, il faut se garder de toute vision à la fois romantique et spontanéiste des événements récents qui ferait fi de l'historicité des mouvements, des registres et des modes de protestation ${ }^{8}$. La mobilisation des Franco-Tunisiens contre l'autoritarisme politique, ici et làbas, n'est pas née avec la Révolution du 14 janvier. Elle doit aussi pour beaucoup au capital contestataire accumulé au fil des années de lutte contre la dictature destourienne ${ }^{9}$. Les "professionnels de la contestation" en immigration ne sont pas des ovnis politiques, des marginaux, des déviants ou des poètes utopistes, mais des pionniers qui ont largement préparé le terrain aux mobilisations de plus en plus collectives de décembre 2010 et janvier 2011. En ce sens, notre analyse s'efforcera d'introduire un arrière-plan historique nécessaire à la compréhension de cette étonnante permanence de l'intérêt des Franco-Tunisiens pour les enjeux politiques du pays d'origine, bien que celui-ci soit resté en "mode silencieux" durant des années de dictature, le silence ne signifiant pas forcément adhésion au régime.

Pour autant, le "moment révolutionnaire"10 doit être analysé en luimême, dans la mesure où il est porteur d'enjeux spécifiques qui ne se réduisent pas à la reproduction mécanique des héritages protestataires du passé. Les passions et les émotions qui se sont exprimées durant la "séquence révolutionnaire" de décembre-janvier sont à l'origine d'une émulation collective des binationaux ${ }^{11}$ totalement inédite en France.

\section{Militances tunisiennes en France avant le 14 janvier : les pionniers}

La tendance des médias, des observateurs profanes et parfois de certains chercheurs est d'analyser les mouvements sociaux pris dans le temps court (le cycle contestation, répression, normalisation), évacuant leur historicité et reproduisant ainsi, consciemment ou inconsciemment, les interprétations romantiques et spontanéistes des acteurs en lutte. Or, sans verser forcément dans une vision déterministe de la militance

8. Cf. TARROW, Sidney ; TILLY, Charles, Politique(s) du conflit : de la grève à la révolution, Paris : Presses de Sciences Po, 2008, $396 \mathrm{p}$.

9. L'adjectif "destourienne" fait référence à la domination sans partage qu'a exercé le Parti socialiste destourien (PSD), devenu en 1987 le Rassemblement constitutionnel démocratique (RCD), sur la vie publique tunisienne durant plus de 50 ans.

10. Cf. ALLAL, Amine ; GEISSER, Vincent, "Tunisie : 'révolution de jasmin' ou intifada ?", Mouvements, $n^{\circ}$ 66, été 2011, dossier "Printemps arabes : comprendre les révolutions en marche", pp. 62-67.

11. Cf. GEISSER, Vincent, "La binationalité en procès : non-dits et hypocrisies d'un débat récurrent", Migrations Société, vol. 23, n 136, juillet-août 2011, pp. 3-10. 
tunisienne en France, qui accréditerait l'hypothèse de la continuité et de la permanence des mouvements sociaux de la diaspora ${ }^{12}$, il nous paraît fécond sur le plan heuristique de faire un détour par l'histoire des combats politiques de l'immigration tunisienne en France. Ces derniers s'inscrivent à la fois dans le prolongement des luttes sociopolitiques du pays d'origine, tout en répondant à des logiques propres à la situation diasporique $^{13}$.

Nous partirons de l'hypothèse que les luttes en contexte migratoire ne constituent pas simplement un "modèle réduit" ou une "antichambre" de celles du pays d'origine, mais relèvent d'une dynamique spécifique, méritant une investigation approfondie.

\section{- L'héritage nationaliste : l'activisme estudiantin tunisien en France}

Contrairement au militantisme algérien dans la France de l'entredeux-guerres, qui était porté à la fois par des prolétaires et des enfants issus de la bourgeoisie indigène (l'Étoile nord-africaine de Messali Hadj est créée à cette époque), l'activisme tunisien dans l'Hexagone se confond presque entièrement avec le mouvement étudiant. Ce prisme estudiantin n'est pas forcément synonyme d'élitisme social, puisque les premiers étudiants tunisiens en France sont plutôt issus de familles de la petite bourgeoisie tunisienne et des régions littorales ayant bénéficié de bourses à leur sortie du collège Sadiki, établissement franco-arabe unique dans son genre qui a formé la plupart des futures élites de l'indépendance ${ }^{14}$. Loin d'être noyés dans la masse des étudiants coloniaux (Indochine, Afrique-Occidentale française, Afrique du Nord, Liban et Syrie mandataires), les étudiants tunisiens se font très vite remarquer par leur détermination dans le combat nationaliste et leur

12. La notion de "diaspora" mériterait sans doute tout un débat théorique qui n’a pas lieu d'être ici. En tout cas, elle est reprise dans les documents officiels des institutions tunisiennes. En 2012, le secrétariat d'État à l'Immigration et aux Tunisiens à l'étranger estimait à environ un million le nombre de Tunisiens inscrits sur les registres consulaires à l'étranger, soit $10 \%$ de la population totale. La grande majorité réside en Europe $(80 \%$, dont 625000 en France, 169000 en Italie, 85000 en Allemagne et 22000 dans les pays du Benelux). Leurs transferts financiers représentent annuellement 1,1 milliard de dinars (500 millions d'euros), dont $70 \%$ sous la forme de transferts monétaires. Les Tunisiens de l'étranger constituent la quatrième source de devises du pays, soit $5 \%$ du PIB et $23 \%$ de l'épargne totale. Source : secrétariat d'État à l'Immigration et aux Tunisiens à l'étranger ; Office des Tunisiens à l'étranger (OTE), 2012.

13. À titre d'éclairage comparatif, voir MEYNIER, Gilbert, "Prolétaires et élites dans les villes françaises dans la première génération de l'émigration algérienne", Les Cahiers de la Méditerranée, n 45, décembre 1992, pp. 173-182.

14. Cf. SRAÏEB, Noureddine, Le collège Sadiki de Tunis, 1875-1956 : enseignement et nationalisme, Paris : Éd. du CNRS, 1997, 346 p. 
sens de l'organisation. C'est notamment eux qui, en 1927, sont à l'origine de la création de l'Association des étudiants musulmans nord-africains (AEMNA) en France, "I au sein de laquelle les militants tunisiens jouent un rôle de premier ordre eu égard à leur nombre et à leur dynamisme. Cette association constitue la pépinière de l'élite politique du mouvement national qui, par la suite, prendra en main les destinées du nouvel État $1{ }^{15}$. Pour l'historien Charles-Robert Ageron, au-delà de sa dimension tunisotunisienne (tous les étudiants d'Afrique du Nord en France avaient le droit d'y adhérer, sauf ceux qui avaient obtenu leur naturalisation, considérés comme traîtres à la cause nationaliste), l'AEMNA a représenté "I une école d'action et de pensée où se préparent les cadres et les méthodes de lutte des nationalismes maghrébins $\|{ }^{16}$.

C'est dans le sillage de l'AEMNA que sera fondé, en 1952, le premier syndicat étudiant tunisien d'obédience séculariste ${ }^{17}$, l'Union générale des étudiants tunisiens (UGET), qui est toujours en activité aujourd'hui. S'il est vrai que l'UGET ne constitue pas exclusivement une organisation de la diaspora, les étudiants émigrés en France jouent un rôle majeur dans sa création et au cours de ses premières années d'existence. Le congrès fondateur du syndicat se déroulera d'ailleurs à Paris (1953), principal lieu où se déroulent les études, mais aussi d'activisme politique des militants tunisiens qui disposent davantage de liberté que sur la scène locale, alors étroitement surveillée par les autorités coloniales. Baignés dans une culture franco-arabe, en contact permanent avec les autres militants estudiantins des organisations nationalistes de l'Empire colonial français (indochinois, algériens, marocains, sénégalais, libanais, etc.), influencés par les intellectuels progressistes du Quartier latin, les étudiants tunisiens exilés à Paris seront les principaux soutiens du Néo-Destour (Parti néo-constitutionnel) et appuieront Bourguiba dans les heures difficiles de la lutte nationaliste: "I'ai milité à la cellule néo-destourienne d'Hammam-Lif [banlieue de Tunis] jusqu'en 1947, c'est-à-dire jusqu'à mon départ pour Dijon et ensuite pour Paris. En juillet 1952, le jour même où j'ai obtenu la licence en droit, des policiers sont venus me demander très courtoisement de quitter la France. Mes camarades français

15. DHIFALLAH, Mohamed, "Bourguiba et les étudiants : stratégie et mutation", in : CAMAU, Michel ; GEISSER, Vincent (sous la direction de), Habib Bourguiba: la trace et l'héritage, Paris: Éd. Karthala ; Aix-en-Provence : CSPC, 2004, pp. 313-324.

16. AGERON, Charles-Robert, "L'Association des étudiants musulmans nord-africains en France durant l'entre-deux-guerres", Revue Française d'Histoire d'Outre-Mer, vol. 70, $\mathrm{n}^{\circ}$ 258-259, $1^{\mathrm{er}}$ et $2^{\mathrm{e}}$ trimestre 1983, pp. 25-55.

17. Avant la création de l'UGET, il existait déjà une organisation étudiante en Tunisie, La Voix de l'étudiant, qui regroupait principalement les étudiants issus de l'Université islamique de la Zitouna. Voir DHIFALLAH, Mohamed, "Bourguiba et les étudiants : stratégie et mutation ", art. cité. 
ont organisé une protestation. Mais le 15 juillet j'ai pris le bateau pour rentrer à Tunis. L'UGET n'existait pas encore. J'ai siégé au bureau de l'AEMNA, dont i'ai été vice-président la dernière année de mes études, en 1952. Mon séjour à Paris a été très important non seulement pour mes études, mais également sur le plan politique. J'ai été chargé de nombreuses missions. Ainsi, par exemple, i'ai représenté le Néo-Destour au Congrès des étudiants arabes à Leyde, en Hollande. Je m'en rappelle très bien parce qu'à l'époque Bourguiba est venu à Paris $11^{18}$.

À peine l'indépendance conquise, l'émulation nationaliste tend progressivement à s'essouffler, accélérant un processus de bureaucratisation des organisations patriotiques, tant en Tunisie que dans les pays d'émigration. L'enjeu principal de l'activisme militant devient la conquête de places au sein de l'appareil d'État, la rhétorique de libération nationale servant souvent à masquer des ambitions personnelles. En même temps, ces tentatives d'instrumentalisation et de monopolisation de la "parole militante" par le nouveau régime issu de l'indépendance suscitent, par effet boomerang, des vocations oppositionnelles qui contribuent à déclencher un nouveau cycle politique de l'activisme tunisien en France. À côté des organisations ouvertement inféodées au régime se structure un espace politique autonome, animé entre autres par des militants de l'émigration.

\section{- La nébuleuse amicaliste en France : émulation patriotique et contrôle autoritaire de l'émigration}

Le fonctionnement du réseau amicaliste tunisien en France n'était guère différent de celui des autres États autoritaires : ses activités et ses projets culturels répondaient moins à une visée philanthropique qu'à une volonté farouche des autorités officielles de maintenir les liens d'allégeance à la mère-patrie au sein des "communautés expatriées", supposées fragilisées par leur situation migratoire. La principale mission de l'Amicale des Tunisiens en France était donc d'entretenir le mythe du retour et de combattre les influences politiques, associatives et syndicales jugées "néfastes" et contraires à l'intérêt national. Selon une logique de mobilisation unanimiste et presque totalitaire, l'allégeance au pays d'origine se confondait avec l'allégeance au régime en place et au parti unique.

18. Entretien avec Béji Caïd Essebsi, ministre de Bourguiba et Premier ministre de la transition démocratique en 2011, réalisé par Michel Camau et Vincent Geisser à Tunis en avril 2002. L'interview a été publiée dans son intégralité dans CAMAU, Michel ; GEISSER, Vincent (sous la direction de), Habib Bourguiba : la trace et l'héritage, Paris : Éd. Karthala ; Aix-en-Provence : CSPC, 2004, 665 p. 
À l'instar des autres États maghrébins (Algérie et Maroc), le pouvoir tunisien a très tôt mis en place des structures associatives pour contrôler politiquement son émigration (face idéologique) et favoriser le rapatriement des devises et des fonds accumulés par les travailleurs tunisiens à l'étranger (face économique). Au départ, la logique d'encadrement politique a prévalu sur toutes les autres, l'Amicale des Tunisiens en France apparaissant comme l'émanation du Parti socialiste destourien (PSD). Bien qu'elle s'affichât officiellement comme une organisation culturelle et apolitique afin de contourner la législation française sur l'obligation de neutralité des associations étrangères (avant l'adoption de la loi du 9 octobre 1981), l'Amicale prenait directement ses ordres à la direction du parti unique, voire, dans certains cas, à la présidence de la République : " En fait, l'Amicale c'était le parti à l'étranger. Nous avions des consignes pour ne pas le crier trop fort, mais c'était la réalité $)^{19}$.

L'Amicale était présente dans toutes les villes françaises où la communauté tunisienne était supposée importante sur le plan numérique. Elle disposait ainsi d'antennes en région parisienne (Paris, Nanterre), en Alsace (Strasbourg), à Lyon, à Grenoble, à Nice, à Marseille et à Toulouse. Les présidents régionaux de l'Amicale étaient directement nommés par le parti unique, choisis parmi les membres les plus dévoués au régime et concurrençant directement les prérogatives des consulats, selon une logique de contrôle idéologique qui s'apparentait à celle de commissaires politiques : " La plupart des présidents, c'étaient des ignorants qui savaient à peine lire et écrire. Ils appliquaient les ordres du parti sans réfléchir $1^{20}$. Distributeurs de "petits services" en tout genre, de passe-droits et d'aides diverses aux émigrés tunisiens en France, les responsables amicalistes se sont progressivement bâtis des réseaux de clientèle qui, bien qu'animés par des motivations purement matérielles, contribuaient à entretenir l'allégeance au parti unique, au régime et à la personne du zaïm (le chef, Bourguiba) : " Nous étions perçus comme des faiseurs de miracles. Chaque fois qu'il y avait un problème, on s'adressait à moi. Je ne pouvais pas circuler en ville sans que quelqu'un me reconnaisse et me demande un service ou une intervention $1{ }^{21}$.

19. Entretien réalisé en 1998 dans le cadre d'une recherche personnelle avec un ancien responsable de l'Amicale des Tunisiens en France qui a tenu à préserver son anonymat, La question polémique des allégeances nationales dans les populations maghrébines de France: l'état des lieux, Institut de recherche sur le Maghreb contemporain (IRMC), Tunis, 1998-1999.

20. Ibidem.

21. Ibidem. 
L'arrivée au pouvoir de Zine el-Abidine Ben Ali ("coup d'État médical" du 7 novembre 1987) se traduit par une reprise en main des structures de l'Amicale, jouant simultanément sur les dimensions culturelle et sécuritaire de l'identification des émigrés et de leurs enfants à leur pays d'origine. Le Rassemblement des Tunisiens de France (RTF), créé de toutes pièces par le nouveau pouvoir, remplace progressivement les anciennes instances amicalistes à bout de souffle, même si les modes de fonctionnement (autoritarisme, paternalisme et surveillance policière des communautés émigrées) restent globalement identiques à ceux de la période précédente. Il est vrai qu'après la chute de Bourguiba les institutions représentatives des Tunisiens à l'étranger vont connaître un certain renouveau. Le régime benaliste, conscient de l'usure des anciennes structures, a encouragé un renouvellement partiel des cadres amicalistes, en faisant appel à de nouvelles compétences plus en phase avec les nouvelles générations issues de l'immigration. L'identité franco-tunisienne, autrefois rejetée au nom de l'exclusivité du lien patriotique, est désormais admise.

De 1987 à 1990, le régime tunisien profite de l'état de grâce suscité par "l'effet du changement" pour mener une politique de séduction à l'égard des intellectuels résidant en Europe. C'est dans la foulée qu'il a fait appel à des diplômés et des universitaires tunisiens de la diaspora qui, jusqu'alors, étaient restés éloignés des organisations officielles prorégime. Durant cette brève période, de nombreux étudiants, universitaires et chercheurs tunisiens choisissent de rejoindre le Rassemblement constitutionnel démocratique ( $R C D$ ) et son émanation directe en France, le Rassemblement des Tunisiens en France : "I Le Rassemblement des Tunisiens en France est officiellement une maison des associations tunisiennes en France. Dans les faits, c'est le siège du RCD - le parti au pouvoir - en France. Du temps où Charles Pasqua était au pouvoir, le régime l'aurait convaincu que pour pouvoir encadrer la population tunisienne en France et lui éviter les risques des infiltrations islamistes, il fallait autoriser l'installation d'un siège officieux du RCD en France. Une ex-résidence universitaire au 36 rue Botzaris 75019 Paris correspondait parfaitement à ce désir. C'est ainsi que le RCD s'est installé en France sous l'appellation Rassemblement des Tunisiens en France. Régie par le statut des associations loi 1901, le Rassemblement des Tunisiens en France a ouvert beaucoup de sections partout en France, et nul ne sait avec quel argent les fonctionnaires qui le peuplent sont payés $11^{22}$.

22. BOUZIDI, Samir, "Un regard calme sur le RCD en France et en Europe", 00216 mag. Le magazine des Tunisiens à l'étranger, 2011, http://www.tunisiensdumonde.com/vous-avez-laparole/2011/01/un-regard-calme-sur-le-rcd-en-france-et-en-europe 
Au tournant des années 1990-2000, face à une recrudescence de la surveillance policière de la diaspora, les Franco-Tunisiens délaissent progressivement les activités associatives orientées vers le pays d'origine, préférant s'investir davantage dans l'espace associatif francofrançais, le champ politique local, ou encore les actions transnationales comme la "cause palestinienne". En effet, nombreux sont les FrancoTunisiens qui ont contribué à organiser des opérations de solidarité avec les territoires palestiniens occupés par Israël (Génération Palestine, Campagne civile internationale pour la protection du peuple palestinien, Secours islamique, etc.). À Paris, Lyon, Grenoble ou Marseille, c'est un sentiment de peur qui domine au sein de la diaspora, et l'évocation des représailles contre les familles restées dans le pays d'origine sert souvent de justification à l'inaction : " Je n'aimais pas Ben Ali, mais je n'osais pas rejoindre l'opposition tunisienne de peur qu'il s'en prenne à ma famille du bled. J'aidais les opposants en sous-main en leur donnant parfois de l'argent et en leur rendant des services $1{ }^{23}$.

Dans la sphère de l'émigration-immigration tunisienne, la dictature de Ben Ali est un sujet tabou : on parle politique mais uniquement en famille ou en présence d'intimes, évitant les lieux publics, notamment les salons de thé et les cafés à narguilé, souvent infiltrés par des agents du régime : " Oui, l'Amicale des Tunisiens nous surveillait. Elle surveillait la moindre de nos activités associatives en France, notamment nous qui étions dans la mouvance musulmane. Les responsables de l'Amicale venaient même voir nos parents pour nous dissuader de participer à telle ou telle activité, nous menaçant de transmettre les informations au consulat $11^{24}$.

Pour autant, il serait réducteur de présenter la diaspora tunisienne en France sous l'angle exclusif de l'apathie politique et du désengagement à l'égard du pays d'origine, d'une part, parce que le silence n'équivalait pas forcément à une adhésion au régime, les critiques à l'égard de la dérive sécuritaire du pouvoir benaliste et des ravages de la corruption de "la famille" (le clan Trabelsi ${ }^{25}$ ) étant fréquentes dans les discussions entre Franco-Tunisiens, même si elles s'énonçaient de manière feutrée ; d'autre part, parce qu'en dépit de la répression cons-

23. Entretien réalisé en février 2011 avec Ahmed N., Français d'origine tunisienne, rédacteur en chef d'un site Web sur la diversité.

24. Wajdi Limam, président de l'UNIT .

25. Le "clan Trabelsi" est la famille de Leila, l'épouse du président Ben Ali, qui s'est livrée à de nombreuses malversations financières durant la dernière partie du règne. Voir BEAU, Nicolas ; GRACIET, Catherine, La régente de Carthage: main basse sur la Tunisie, Paris : Éd. La Découverte, 2009, 178 p. 
tante exercée par les "services" tunisiens sur le territoire français ${ }^{26}$, cela n'a jamais empêché un militantisme autonome de se développer en marge des organisations officielles et de préparer ainsi le terrain à un engagement plus massif des Franco-Tunisiens dans les mouvements contestataires.

\section{- Sociabilités gauchistes et tiers-mondistes en diaspora : de l'opposition radicale au régime à l'associationnisme immigré}

L'émergence des sociabilités de gauche en diaspora trouve son origine dans les débats contradictoires qui ont agité les milieux estudiantins tunisiens au cours des années 1960-1970 27 et qui se sont traduits par une effervescence militante sans précédent dans l'histoire politique de l'émigration-immigration. Au départ, la quasi-totalité des étudiants politisés adhèrent à l'UGET, syndicat certes unique, mais composé de nombreux courants, allant de l'extrême gauche marxiste-léniniste aux communistes orthodoxes, en passant par les maoïstes, les trotskistes et les travaillistes ${ }^{28}$, sans oublier les étudiants destouriens qui soutiennent directement les orientations du régime.

Les étudiants tunisiens de l'Hexagone sont à la fois nourris de l'influence des intellectuels français (Jean-Paul Sartre, Henri Lefebvre, Louis Althusser...), mais aussi très largement des discussions passionnées sur l'avenir du tiers-monde qui agitent les milieux asiatiques, africains et maghrébins exilés en France : " À Paris, je fréquentais beaucoup l'entourage de Sartre à Saint-Germain, je lisais la revue Les Temps Modernes. Je le voyais en personne, car il était accessible, il se produisait devant des auditoires d'étudiants, notamment d'étudiants maghrébins. Je savais qu'il sympathisait avec Kateb Yacine et cela me le rendait encore plus proche. La polémique avec le Parti communiste m'interpellait aussi : elle me paraissait porteuse de richesse. Cet homme engagé qui contestait ce

26. Les consulats de Tunisie en France ont connu une inflexion sécuritaire très nette sous la période Ben Ali. Outre des consuls ou vice-consuls directement recrutés parmi les cadres du ministère de l'Intérieur, les consulats disposaient d'antennes sécuritaires chargées de suivre les activités politiques et associatives des Franco-Tunisiens, voire, dans certains cas, d'exercer des pressions et des menaces à l'encontre des individus et des familles. GEISSER, Vincent, enquête personnelle.

27. Cf. BÉCHIR-AYARI, Michaël, S'engager en régime autoritaire : gauchistes et islamistes dans la Tunisie indépendante, op. cit.

28. II existe en Tunisie un courant travailliste issu de la tradition syndicale ouvrière et représenté par I'Union générale tunisienne du travail (UGTT) et son leader Ahmed Ben Salah. Voir HAMZAOUI, Salah, "Champ politique et syndicalisme en Tunisie", Annuaire de l'Afrique du Nord, vol. 38, 1999, pp. 369-380. 
qu'il y avait de totalitaire dans la pensée marxiste me paraissait intéressant, alors qu'à l'époque je n'avais pas lu une seule ligne de Marx. C'est plus tard que i'ai commencé à découvrir le marxisme à travers les citations de Sartre. J'ai commencé à lire les ouvrages de Rosa Luxembourg, de Jaurès et tout ce qui pouvait paraître aux éditions Maspéro. Mais Marx et Lénine, je ne les ai lus qu'après mon retour à Tunis. Les deux livres qui m'ont le plus marqué étaient Le Manifeste du Parti communiste ef Le 18 Brumaire de Louis Bonaparte. J'ai été aussi influencé par les milieux militants algériens, parce qu'ils avaient un discours tiers-mondiste sur l'industrialisation, l'agriculture, la réforme agraire... Je me rappelle que je lisais régulièrement Révolution africaine et les papiers de Mohammed Harbi que je n'ai d'ailleurs jamais rencontré. J'ai été marqué également par le leader marocain Mehdi Ben Barka qui est venu à plusieurs reprises nous rencontrer au 115 boulevard Saint-Michel : son discours me plaisait. II faut dire que les débats idéologiques sur le Maghreb me concernaient beaucoup $1{ }^{29}$.

Dans cette agitation entre factions étudiantes, Paris occupe sans aucun doute une position spécifique : la section parisienne de l'UGET est très rapidement dominée par les opposants de gauche qui choisissent d'affronter directement les étudiants destouriens restés fidèles à Bourguiba et au PSD, le parti unique, l'enjeu étant bien sûr le contrôle de la direction de la centrale syndicale. Dans ce contexte d'affrontements idéologiques est fondé au tout début des années 1960 le Groupe d'études et d'action socialistes en Tunisie (GEAST), plus connu sous le nom de groupe Perspectives, qui est le titre de sa revue, imprimée en France et diffusée clandestinement en Tunisie.

Bien qu'animé principalement par des étudiants et des intellectuels, le groupe Perspectives sera aussi à l'origine d'initiatives en milieu immigré et prolétaire visant à conscientiser les ouvriers à la lutte contre l'autoritarisme du régime; en 1969, l'organisation lance une publication, El Amal Ettounsi (L'ouvrier tunisien), qui a la particularité d'être rédigée non pas en arabe littéraire, langue de l'élite, mais en dialecte tunisien (darija), la langue du peuple, et donc de rendre accessible la rhétorique de la cause révolutionnaire aux travailleurs immigrés. C'est précisément de cette mouvance que vont naître les organisations tunisiennes de soutien aux émigrés-immigrés au milieu des années 1970, leurs militants évo-

29. Entretien avec Nouredine Ben Kheder, ancien leader de l'extrême gauche tunisienne, condamné à 16 ans de prison sous Bourguiba, réalisé par Michel Camau et Vincent Geisser à Tunis en avril 2002. L'interview a été publiée dans son intégralité dans CAMAU, Michel; GEISSER, Vincent (sous la direction de), Habib Bourguiba : la trace et l'héritage, op. cit. 
luant progressivement de la "lutte révolutionnaire" (faire tomber le régime) aux actions de solidarité avec les immigrés ${ }^{30}$. Cette conversion s'explique par diverses raisons qu'il serait trop long de développer dans le cadre de cette contribution, mais disons brièvement qu'elle est surtout le produit d'une trajectoire migratoire qui a vu une grande partie des anciens étudiants tunisiens "se fixer" définitivement en France, en se distanciant progressivement des enjeux politiques du pays d'origine. En l'espace de quelques années (1970-1980), les activistes de l'extrême gauche tunisienne basés dans l'Hexagone vont ainsi reconvertir leur capital militant accumulé durant des années de lutte contre la dictature en capital associatif axé sur la "cause immigrée".

Cette trajectoire est typique d'une organisation comme la Fédération des Tunisiens pour une citoyenneté des deux rives (FCR), fondée en 1974 sous le nom d'Union des travailleurs immigrés tunisiens (UTIT), animée par des anciens de la gauche tunisienne qui se sont progressivement impliqués sur le terrain de l'immigration et notamment dans la "cause des sans-papiers" et, plus récemment, dans les questions de citoyenneté et de lutte contre les discriminations : "I En 1994, après une longue période de réflexion et de débat, l'association a décidé de modifier ses statuts pour prendre le nom de Fédération des Tunisiens pour une citoyenneté des deux rives (FTCR), officialisant son cheminement vers un ancrage dans la société française tout en marquant un attachement au pays d'origine et soulignant la centralité de la question de citoyenneté dans ses valeurs. La Fédération a mis l'accent sur un important travail de réflexion sur les thèmes de laïcité, citoyenneté, étrangers en situation irrégulière, et a été une des premières associations à s'interroger sur la place de l'islam en France $11^{31}$.

II faut se garder toutefois d'une représentation linéaire de l'évolution des trajectoires militantes en contexte migratoire. Car, s'il est vrai que dans un premier temps les militants tunisiens de l'immigration ont eu tendance à se distancier des enjeux politiques du pays d'origine - notamment dans les premières années du règne de Ben Ali ils les ont progressivement réinvestis, au point que la FTCR peut être considérée comme l'une des principales actrices de l'opposition tunisienne en France contre la dictature benaliste. En 2008, par exemple, la FTCR jouera un rôle majeur dans la révolte du bassin minier de Gafsa (prélude à la Révolution du 14 janvier), en relayant directement les informations sur la mobilisation sociale et la répression policière, alors

30. C'est le cas notamment de Saïd Bouziri, décédé en 2009, auquel nous rendons hommage.

31. Présentation de la FTCR par elle-même, rubrique "La FTCR", www.citoyensdesdeuxrives.eu/better 
que celles-ci étaient inaccessibles depuis la Tunisie : II II est fort probable que, sans l'action d'une organisation comme la FTCR, la révolte du bassin minier n'aurait pas eu l'écho international qu'elle a connu, le ministère de l'Intérieur ayant "cadenassé" tous les moyens d'information et de communication, à tel point que les Tunisiens de France étaient souvent davantage au courant de ce qui se passait réellement à Redeyef [épicentre du mouvement social] que les Tunisiens de l'intérieur eux-mêmes $11^{32}$.

\section{- Exils islamistes en France : précaires et entrepreneurs de la "cause musulmane"}

Les étapes de l'installation des exilés islamistes tunisiens en Europe correspondent aux trois grandes vagues de répression: juin 1981 (démantèlement des structures du Mouvement de la tendance islamique $^{33}$ ) ; 1986-1987 (intensification des arrestations et des condamnations à la fin du règne de Bourguiba) et 1991-1992 (grande répression sous Ben Ali avec plusieurs milliers d'arrestations dans les rangs des militants de l'islam politique) ${ }^{34}$. Après avoir tenté de séjourner dans les pays arabes voisins (Algérie, Maroc, Libye, Soudan, etc.), de nombreux islamistes se sont retrouvés II à bord des filières d'émigration clandestine à travers la Méditerranée $\|^{35}$ et ont ainsi débarqué en France entre 1991 et 1996, via l'Italie ou l'Espagne, après un séjour plus ou moins bref au Maghreb. Parmi eux figuraient de nombreux étudiants, mais également de petits artisans et commerçants ${ }^{36}$.

D'autres encore vont choisir Londres, lieu d'asile et d'activisme politique pour de nombreuses organisations arabes appartenant à la nébuleuse islamiste. Par exemple, le président-fondateur du parti islamiste

32. CHOUIKHA, Larbi ; GEISSER, Vincent, "Retour sur la révolte du bassin minier : les cinq leçons politiques d'un conflit social inédit”, L'Année du Maghreb [En ligne], VI | 2010, consulté le 7 juillet 2012, http://anneemaghreb.revues.org/923

33. Le Mouvement de la tendance islamique (MTI) est l'ancêtre d'Ennahda (Renaissance), le parti islamiste tunisien. Pour davantage d'informations sur le mouvement islamiste tunisien, voir BURGAT, François, L'islamisme au Maghreb : la voix du Sud, Paris : Éd. Karthala, 1988, 304 p. ; CAMAU, Michel ; GEISSER, Vincent, "L'islamisme imaginaire : identité obsédante et structurante des scènes politiques tunisiennes?", Maghreb-Machrek, n 175, printemps 2003, pp. 35-52.

34. C'est durant cette période que les ONG telles qu'Amnesty International ou la Fédération internationale des droits de l'homme $(\mathrm{FIDH})$ recensent une cinquantaine de décès sous la torture.

35. TOSCANE, Luiza, Terre d'écueil, le droit d'asile benaliéné, http://www.reveiltunisien.org/ article.php3?id_article $=2373$

36. BÉCHIR-AYARI, Michaël, "Rester le même tout en devenant un autre : les 'islamistes' tunisiens exilés en France", Maghreb-Machrek, n 194, hiver 2007-2008, dossier "Les mouvements islamistes des armes aux urnes", pp. 55-73. 
tunisien Ennahda, Rached Ghannouchi, après avoir tenté de s'installer en France, faute d'autorisation du ministère de l'Intérieur ${ }^{37}$ sera contraint de se replier sur la Grande-Bretagne où il deviendra un leader respecté parmi les communautés musulmanes locales. Sur ce plan, il convient de revenir à notre hypothèse de départ, en soulignant l'influence des contextes migratoires sur les modes de mobilisation politique. En effet, une brève comparaison entre le milieu des exilés islamistes tunisiens à Londres et à Paris permet de mettre en évidence le "poids" de la société du pays d'immigration et les différentes formes d'insertion économique, sociale et politique sur les manières de concevoir le militantisme en diaspora.

En Grande-Bretagne, l'insertion des exilés tunisiens va se jouer principalement au sein de la "mouvance islamique" proche et moyenorientale qui est déjà bien implantée dans la capitale du royaume. Les islamistes tunisiens deviendront très vite des acteurs islamiques transnationaux, profitant de la présence à Londres des grands médias arabophones (chaînes satellitaires et presse écrite) pour rayonner sur l'ensemble de l'umma islamiya (la communauté islamique mondiale). En France, les mobilisations politiques des exilés islamistes se dessineront selon des lignes légèrement différentes, davantage "intégrationnistes". D'une part, les islamistes tunisiens côtoient régulièrement les autres opposants politiques, y compris ceux de la gauche laïque et des anciens bourguibiens, avec lesquels ils développent une forte conscience de la tunisianité : " Nous sommes Tunisiens avant d'être islamistes ". II n'est pas rare qu'ils organisent des réunions transpartisanes, où tous, leaders et militants de l'opposition en exil, se retrouvent, y compris ceux connus pour leurs positions laiiques. D'autre part, ils vont s'impliquer plus activement dans le paysage islamique français au sein duquel ils deviennent très rapidement des acteurs de référence. Au début des années 1990, les premières institutions éducatives musulmanes en France sont souvent portées par d'anciens islamistes tunisiens qui prônent à la fois l'intégration à la société française et la préservation de l'identité musulmane des enfants de l'immigration. Parce qu'ils ont souvent la réputation d'être davantage éduqués et lettrés que la moyenne des cadres de l'islam de France (niveau d'études universitaires acquis dans le pays d'origine), les exilés islamistes tunisiens acquièrent très vite une position dominante dans le paysage islamique hexagonal et sont à

37. Entre 1993 et 1997, le ministre de l'Intérieur Charles Pasqua renforce la collaboration avec les autorités sécuritaires tunisiennes, afin que la France ne devienne pas une "base-arrière" et un "lieu d'activisme politique" pour les islamistes tunisiens. 
l'initiative de la plupart des institutions de formation et des futurs établissements scolaires privés musulmans (école primaires, collèges et lycées) ${ }^{38}$.

Cependant, cette influence des islamistes tunisiens sur la socialisation des Français héritiers de l'immigration tunisienne doit être largement nuancée. D'abord, parce qu'elle ne touche qu'une minorité de jeunes croyants et pratiquants actifs dans les mosquées et les organisations islamiques. En effet, la grande majorité des descendants de migrants tunisiens ne fréquente pas les institutions islamiques de l'Hexagone. Ensuite, les islamistes tunisiens observent une relative neutralité politique dans la gestion de leurs institutions éducatives, condition sine qua non de leur acceptation par les autorités françaises. Ces institutions s'adressant à un public assez hétéroclite (des musulmans français de toutes origines) ${ }^{39}$, la "question tunisienne" est donc rarement abordée dans les cours, si ce n'est de manière anecdotique. Enfin, et c'est peut-être la raison principale, à l'instar des anciens militants d'extrême gauche, les islamistes tunisiens véhiculent une manière de faire de la politique dans laquelle la plupart des Franco-Tunisiens ne se reconnaissent pas. Si les enfants héritiers de l'immigration sont sensibles aux aspects humanitaires et reconnaissent aux exilés islamistes le statut de "victimes de la dictature", ils partagent rarement leurs perceptions et leurs conceptions du militantisme. Le conflit larvé blédards/banlieusards ${ }^{40}$, à propos des organisations tunisiennes laïques, s'applique également à la mouvance islamiste qui connaît des difficultés comparables à mobiliser les nouvelles générations sur le dossier tunisien.

De ce fait, on peut comprendre que ni les organisations militantes tunisiennes issues de l'extrême gauche (FTCR, UGET-France, Associations

38. Parmi les institutions créées directement ou indirectement par des exilés islamistes tunisiens, on peut citer le collège-lycée musulman La Réussite à Aubervilliers, le collège Éducation \& Savoir à Vitry-sur-Seine, Connaître, comprendre et vivre l'islam (CERSI) à Saint-Denis, la branche parisienne de I'Institut européen des sciences humaines (IESH), I'Institut Formation Avenir à La Courneuve, l'Institut international de la pensée islamique (IIIT) à Saint-Ouen, l'Institut français d'études et de sciences islamiques (IFESI) à Boissy-Saint-Léger, le collège musulman Ibn Khaldoun à Marseille, etc. Source : enquête personnelle.

39. Notre recherche de terrain sur les institutions éducatives islamiques en région parisienne et à Marseille tend à montrer un public très divers par ses origines nationales (enfants de Marocains, Algériens, Tunisiens, Sénégalais, Comoriens, convertis, etc.), reflétant grosso modo les caractéristiques sociologiques de la jeunesse des quartiers populaires.

40. Cf. SCHIFF, Claire, "Beurs et Blédards : une dichotomie 'indigène' à usages multiples. Rapports de genres, de classes et de culture dans les forums de discussions en ligne dédiés à la communauté maghrébine", communication au colloque "Catégorisation(s) et migrations" organisé par le CERI-Science Po le 14 mars 2008, http://migractions.free.fr/Textes\%20colloque/ Schiff\%20Claire.rtf 
des Tunisiens en France, etc.) ni celles issues de la mouvance islamiste ${ }^{41}$ ne joueront un rôle-clé durant le "moment révolutionnaire" de décembre 2010 et janvier 2011. Si elles ont incontestablement préparé le terrain à la mobilisation et surtout à la conscientisation à l'égard des enjeux politiques du pays d'origine, elles n'en ont pas été les acteurs centraux.

\section{Le "moment révolutionnaire" : de l'unanimisme patrio- tique aux recompositions conflictuelles de l'espace franco-tunisien}

Les premières semaines après la fuite de Ben Ali et l'enclenchement d'un processus de transition politique en Tunisie ont été l'occasion d'un foisonnement d'activités politiques en tout genre, empruntant généralement un registre unanimiste, conforme à l'image d'Épinal d'une "révolution populaire" contre le régime autoritaire. Cette ambiance patriotique s'est très largement retrouvée en contexte migratoire, où les opposants traditionnels à la dictature - islamistes, anciens activistes de gauche et bourguibistes - ont communié avec ces milliers de FrancoTunisiens anonymes qui, "révolution" faisant, se découvraient une vocation politique à éradiquer les restes de l'autoritarisme et à œuvrer à l'instauration de la démocratie dans le pays d'origine. Ainsi, entre janvier et juin 2011 , nous avons pu recenser plus d'une centaine d'initiatives portées par les héritiers de l'immigration tunisienne en France allant de la création ex nihilo d'associations de solidarité avec la "révolution tunisienne" à la mise en place d'initiatives humanitaires pour soutenir les régions sinistrées et venir en aide aux réfugiés à la frontière tunisolibyenne, en passant par l'organisation de colloques, séminaires, tables rondes ou le lancement de blogs racontant les exploits des révolutionnaires tunisiens. Ce climat d'unanimisme entraîne une recomposition partielle des sociabilités associatives et politiques de la diaspora, les Franco-Tunisiens délaissant temporairement leur mobilisation associative et leur affiliation politique habituelles pour consacrer l'essentiel de leur militance à la "cause tunisienne".

41. En dehors des organisations éducatives et culturelles musulmanes, le mouvement associatif islamiste tunisien en France était représenté avant le 14 janvier 2011 par l'organisation Solidarité tunisienne dont le siège social était à Aubervilliers. Aujourd'hui, la mouvance associative islamiste dans l'Hexagone connaît un certain renouveau, notamment à travers l'association Tunisiens des deux rives (T2RIV) dont le slogan est "Tunisie, investir, s'investir », http://www.t2riv.org 


\section{- Une conscientisation très ordinaire : la famille proche contre l'État despote}

Dans le cadre d'une telle analyse des mobilisations des FrancoTunisiens durant la "séquence révolutionnaire" de décembre 2010janvier 201 1, il faut se garder de deux types d'interprétation véhiculés fréquemment par les discours issus du sens commun : d'une part, celle d'une diaspora qui, en quelques semaines, serait passée de l'état d'apathie politique, voire du soutien tacite au régime dictatorial, à un ralliement opportuniste au mouvement révolutionnaire; d'autre part, celle d'une diaspora fortement politisée et conscientisée qui, à l'instar du peuple tunisien, attendait des circonstances favorables pour passer à l'acte de rébellion contre le "système Ben Ali".

Outre leur caractère globalisant, ces deux visions de l'engagement des Franco-Tunisiens dans les mouvements protestataires de l'hiver 2010-2011 nous semblent faire fi des modes de politisation ordinaires, presque domestiques, qui échappent très largement aux canaux classiques de la mobilisation politique. Si le choix de la prudence et du silence était l'attitude la plus fréquemment adoptée au sein de la diaspora par peur des représailles sur la famille vivant en Tunisie, les discours critiques sur le régime de Ben Ali et la dérive maffieuse de sa famille circulaient très largement depuis plusieurs années dans les milieux de l'immigration tunisienne en France, au point de devenir l'un des sujets de conversation quotidien des Franco-Tunisiens. À l'inverse, les discours de soutien au régime autoritaire étaient plutôt rares, y compris chez ceux qui avaient pu, au début des années 1990, croire au "miracle réformateur" du nouveau président ${ }^{42}$.

Ces dernières années, la littérature de l'opposition au régime et les opuscules clandestins rédigés par des élites dissidentes sur "l'état réel" de la Tunisie connaissaient une très large diffusion au sein de la diaspora franco-tunisienne. Plus que la dérive sécuritaire du régime Ben Ali, qui était davantage un sujet d'"opposants professionnels", c'étaient les thèmes de la corruption et du népotisme qui alimentaient les conversations familiales et amicales au sein de l'immigration et qui contribuaient à susciter une forme de "conscience rebelle", certes fluide, mais bien réelle. Un sociologue qui aurait analysé superficiellement les attitudes et

42. Il est vrai que, au milieu des années 1990 , on pouvait encore rencontrer au sein de la diaspora ce type de discours : " Depuis que Ben Ali est arrivé au pouvoir, on traite mieux les immigrés. Il paraît qu'il a donné des consignes aux douaniers et aux policiers pour mieux accueillir les immigrés pendant l'été. D'ailleurs, grâce à Ben Ali, la Tunisie a beaucoup changé ". Entretien avec Hamid M., sans-papiers tunisien, réalisé à Montpellier en 1996. 
les comportements des Franco-Tunisiens quelques semaines avant la Révolution du 14 janvier aurait pu conclure à la démobilisation totale ou, pire, à une posture de soumission volontaire au régime ${ }^{43}$, donnant l'image d'une diaspora tunisienne relativement satisfaite de la politique officielle de l'État d'origine.

L'absence de mobilisations visibles et massives contre la dictature au sein de l'immigration tunisienne en France - les manifestations antiBen Ali étaient rares et squelettiques ${ }^{44}$ - donnait l'impression d'une acceptation complice, sinon passive, des règles du jeu édictées par le "système Ben Ali" à l'égard de sa diaspora : la tranquillité toute exotique du retour estival dans le pays d'origine, avec quelques avantages fiscaux accordés aux émigrés en échange de leur silence. Pourtant, audelà de cette image trompeuse de soumission volontaire au régime, on pouvait percevoir au sein de la diaspora la montée d'un mécontentement diffus. Certes, il ne se traduisait pas par une "entrée en résistance" massive des Franco-Maghrébins (vision romantique de l'engagement), mais par des mouvements d'humeur, des contre-conduites et des microactes d'insubordination (le fait, par exemple, de ne plus s'inscrire sur les listes consulaires, de refuser de participer aux élections truquées, de boycotter Canal 7, la chaîne nationale diffusée par satellite) révélateurs d'une très nette désaffection de la diaspora à l'égard du système. Sur ce plan, les sociabilités familiales et amicales ont joué un rôle tout aussi déterminant dans la conscientisation des Franco-Tunisiens que les modes institutionnalisés d'opposition : "la petite histoire" rapportée par un proche parent du bled sur la corruption, les vexations quotidiennes de la police, les passe-droits pratiqués par les caciques locaux du RCD (le parti présidentiel), les fastes de "la famille" (Ben Ali-Trabelsi) ont contribué à accélérer "à distance" ce processus de délégitimation du régime et de démystification de sa toute-puissance.

On comprend dès lors les raisons pour lesquelles la diaspora tunisienne en France a pu basculer dans le "camp de la révolte", refusant de cautionner la propagande du pouvoir (selon laquelle les protes-

43. Cf. HIBOU, Béatrice, La force de l'obéissance : économie politique de la répression en Tunisie, Paris : Éd. La Découverte, 2006, 362 p.

44. Notre observation longitudinale des mouvements anti-régime en diaspora, conduite sur plus de 10 ans (2000-2010), tend à montrer la faiblesse des mobilisations publiques, qui regroupaient moins d'une centaine de personnes, parmi lesquelles des opposants de la gauche radicale, des islamistes et des sympathisants français de la "cause tunisienne". Les Franco-Tunisiens étaient, eux, quasiment absents de ces manifestations contre le régime Ben Ali. II a fallu attendre fin décembre 2010 (quelques jours avant la fuite du président) pour que les héritiers de l'immigration tunisienne en France osent franchir le pas de la contestation dans l'espace public. 
tations de Sidi Bouzid et de Kasserine ${ }^{45}$ seraient le fait d'islamistes et de terroristes ${ }^{46}$ ) et de soutenir finalement les mouvements protestataires de l'hiver 2010-2011. En somme, ce ralliement aux mobilisations antirégime est le fruit d'un processus relativement long de désaffection et de désaffiliation à l'égard de la "politique officielle" (" Ben Ali ne nous représente plus ", "les Trabelsi nous font honte ") entamé bien avant les événements révolutionnaires du 14 janvier.

\section{- Les continuateurs : la difficile reconversion du capital résistant en capital participatif}

Mettant en avant leur rôle de "résistants" au régime de Ben Ali, les organisations issues de la gauche indépendante et de la mouvance islamiste se sont imposées de facto dans la diaspora comme les références morales et politiques de la transition démocratique. II est vrai que leur statut d'opposants historiques à la dictature leur a donné une certaine aura auprès des immigrés tunisiens et de leurs descendants binationaux qu'elles ont tenté de mettre à profit pour imposer leur leadership dans l'espace sociopolitique de la diaspora. Bien sûr, les anciens gauchistes reconvertis dans le secteur associatif et les exilés islamistes ont emprunté des voies différentes. Les premiers ont surtout capitalisé leur expérience oppositionnelle pour conforter leurs relations auprès de leurs partenaires français (Ligue des droits de l'homme, Amnesty international, syndicats de salariés, Parti communiste français, Parti socialiste, Europe Écologie Les Verts, Front de gauche, les ONG de solidarité avec l'immigration, etc.) et apparaître ainsi comme les experts de la "question tunisienne" en France. Les seconds se sont davantage attelés à préparer leur "retour" dans le pays d'origine (la majorité d'entre eux l'avaient quitté depuis plus de 20 ans), tout en cherchant à reconstruire les bases de l'islamisme partisan dans l'Hexagone (parti Ennahda), en s'appuyant notamment sur les organisations de l'islam de France qui comptent dans leurs rangs de nombreux Franco-Tunisiens.

Ces stratégies de reconversion partielle du "capital résistant" en "capital participatif" ont été couronnées de plus ou moins de succès. Une organisation comme la FTCR, par exemple, est devenue en quelques

45. Villes du centre-ouest qui ont été les épicentres de la contestation de l'hiver 2010-2011.

46. Propagande qui était notamment martelée dans les médias français et européens par l'ambassadeur de Tunisie à l'UNESCO, Mezri Haddad, visant à discréditer les manifestants en les faisant passer pour des disciples d'Al-Qaïda. 
semaines l'interlocuteur incontournable des acteurs associatifs, politiques et syndicaux français sur la "question tunisienne", position avantageuse qui a permis à certains militants de l'immigration d'envisager des carrières politiques et institutionnelles dans le pays d'origine. Le "capital résistant" accumulé durant des années de luttes contre la dictature parmi la diaspora a été à l'origine de nouvelles vocations et de mobilités ascendantes au sein des institutions de la transition démocratique en Tunisie.

Dans la galaxie islamiste, on a pu assister à des reconversions similaires : de nombreux réfugiés, précarisés par l'exil, ont pu ainsi envisager une deuxième vie dans le pays d'origine, alors que quelques semaines avant la chute de Ben Ali la plupart d'entre eux pensaient mourir sans jamais revoir la mère patrie. Chez les élites de la mouvance islamiste, le projet de retour s'est également accompagné d'un projet de reconstruction partisane : les anciens dirigeants du parti Ennahda, en exil en France et en Grande-Bretagne, ont été les principaux artisans du retour en force du parti islamiste sur la scène politique et électorale en Tunisie : "I certains étaient RMIstes avant le 14 janvier. Quelques semaines après, ils sont devenus ministres ", ironise-t-on dans certains cercles politiques tunisiens hostiles au nouveau gouvernement de coalition dominé par les islamistes ${ }^{47}$.

Si l'image de "résistants" ou d"'opposants historiques" à la dictature a incontestablement permis aux anciens de la gauche associative et aux exilés islamistes d'acquérir un véritable prestige au sein de la diaspora tunisienne en France, elle ne s'est pas forcément traduite par la captation massive de nouveaux adhérents et militants parmi les binationaux ${ }^{48}$ qui éprouvent toujours des difficultés à se reconnaitre dans les organisations historiques, préférant se mobiliser parallèlement.

47. Aux élections du 23 octobre 2011, les islamistes du parti Ennahda ont remporté la majorité relative des sièges à l'Assemblée constituante, devenant ainsi les acteurs-clés du nouveau gouvernement de transition. Parmi les nouveaux élus, on recense de nombreux anciens exilés en France et dans les pays européens, dont certains étaient candidats à titre "émigré", et d'autres dans le cadre de circonscriptions internes au territoire tunisien. On citera comme exemple emblématique le cas de Meherzia Labidi, aujourd'hui vice-présidente de l'Assemblée constituante, qui a passé les 20 dernières années de sa vie en France.

48. Si aux élections de l'Assemblée constituante le parti islamiste Ennahda est arrivé en tête dans les circonscriptions françaises (France 1 et France 2) en obtenant environ $30 \%$ des suffrages, il n'a pas enregistré pour autant un afflux massif de nouveaux militants. Quant à la gauche associative présente sur plusieurs listes, elle a essuyé un échec cinglant sur le plan électoral, à l'instar de Tarek Ben Hiba, président de la FTCR et tête de liste des Associatifs de l'immigration tunisienne, pourtant connu depuis de nombreuses années pour son engagement pour les libertés en Tunisie, qui n'a même pas réussi à se faire élire. En dehors des islamistes, les seuls partis à tirer leur épingle du jeu électoral sont le Congrès pour la République (CPR) de Moncef Marzouki et le Forum démocratique pour le travail et les libertés (FDTL) de Mustapha Benjaafar, partis classés au centre gauche mais refusant de verser dans l'anti-islamisme. 


\section{- Les initiateurs d'une "relation nouvelle": un travail de mise en cohérence identitaire}

La chute annoncée du régime de Ben Ali a suscité au sein de la diaspora un processus de libération de la parole sans précédent dans l'histoire de l'immigration tunisienne en France. Des initiatives diverses portées par des Franco-Tunisiens ont foisonné de toute part, souvent sans lendemain, révélant le caractère labile et incertain des contextes de transition politique. De nombreux binationaux se sont ainsi trouvés une vocation politique pour le pays d'origine, se réinventant au passage une histoire militante, sous le registre récurrent de la "résistance silencieuse" au benalisme : " Nous ne parlions pas, mais nous agissions $)^{49}$.

Ce processus de reconstruction de l'engagement diasporique sous la dictature est d'ailleurs comparable à ce qu'a pu connaître la France au moment de la Libération : le mythe résistancialiste a rempli une fonction de cohésion nationale, véhiculée autant par les "vrais" résistants que par les "tièdes", désignant sur un mode passionnel les boucs émissaires et les figures expiatoires (les "collabos") pour mieux faire oublier le silence de la majorité. Le "syndrome Ben Ali" ne fait que commencer $^{50}$. La chasse aux cadres du RCD (parti quasi unique), la mise au pilori des personnalités de l'immigration les plus impliquées avec l'ancien système et la dénonciation de la collaboration de l'État français avec la dictature de Ben Ali deviennent les grands thèmes de discussion chez les Franco-Tunisiens, organisant dans la foulée colloques et tables rondes, rassemblements devant les consulats honnis ou montant des opérations "coup de poing" pour chasser les derniers suppôts du benalisme encore présents sur le territoire français. Les opposants d'hier, que l'on osait à peine fréquenter au temps de la dictature, deviennent temporairement des "héros" que l'on s'arrache dans les différentes réunions des Tunisiens de France qui entendent être reconnus tant dans leur rôle de résistants ("I nous aussi, nous avons contribué à la chute de Ben Ali II) que comme acteurs centraux de l'édification de la future démocratie (II nous voulons notre place dans la transition politique 11$)$.

Ce qui est intéressant pour le sociologue de l'immigration, ce ne sont pas tant les énoncés opportunistes, qui sont le fait de tout processus de "reconstruction politique" après un événement majeur (révolution,

\footnotetext{
49. Expression que nous avons pu relever lors de nombreuses discussions avec les FrancoTunisiens sur leur rôle sous la dictature.

50. Cf. ROUSSO, Henry, Le syndrome de Vichy de 1944 à nos jours, Paris : Éd. du Seuil, 1990, 414 p.
} 
libération nationale, chute d'une dictature, etc.), que le travail de mise en cohérence identitaire opéré par les individus. Or, après le 14 janvier 2011 , il s'est produit au sein de la diaspora tunisienne en France un processus de recréation identitaire sur lequel il convient de s'arrêter brièvement afin de ne pas céder trop facilement aux clichés romantiques et résistancialistes. La "tunisianité révolutionnaire", revendiquée haut et fort par les Franco-Tunisiens après la chute de Ben Ali, est censée constituer le ciment commun de toutes les actions en direction du pays d'origine. En effet, elle s'affiche sans complexe dans la plupart des plateformes associatives franco-tunisiennes rédigées dans le contexte de la transition démocratique.

Toutefois, le registre unanimiste, que l'on pourrait identifier à un II patriotisme diasporique ${ }{ }^{51}$, réactivé par le contexte d'ébullition révolutionnaire, parvient difficilement à surmonter les clivages migratoires, sociaux et politiques qui trouvent dans ce foisonnement associatif de l'après-Ben Ali un nouveau terrain d'expression. Car, loin du cliché de la "génération spontanée", la majorité des Franco-Tunisiens qui décident de s'engager sont généralement des militants politiques et associatifs binationaux, issus des nouvelles classes moyennes de l'immigration (éducateurs, enseignants, fonctionnaires, professions libérales, etc.) et qui ont déjà une solide expérience des mobilisations. La plupart d'entre eux, s'ils n'avaient jamais milité pour la cause tunisienne par peur de la police politique, ont fait leurs premières armes dans les luttes contre les discriminations raciales en France (dénonciation de l'islamophobie et du racisme anti-arabe) et au sein de la mouvance pro-palestinienne. Certains de ces "nouveaux militants" de la cause tunisienne sont même des élus de la République, détenteurs d'un mandat dans une collectivité locale en France (conseil municipal, conseil régional, etc.).

Contrairement à une idée reçue, ces militants de la cause tunisienne ne sont donc pas des nouveaux venus de l'activisme associatif et politique. Leur habitus militant a certes constitué un facteur de cohésion du groupe, mais a été aussi source de nombreuses frictions avec d'autres profils de Tunisiens engagés. D'abord, avec les étudiants blédards qui ont vu d'un très mauvais œil le leadership des banlieusards (II comment ces jeunes qui ne parlent même pas l'arabe et qui ne connaissent pas leur pays peuvent revendiquer une expertise sur la Tunisie ? $11^{52}$ ), mais aussi avec

51. GEISSER, Vincent ; KELFAOUI, Schérazade, "La nation d'origine réinventée : la persistance du 'mythe national' chez les Français originaires du Maghreb", Confluences Méditerranée, $n^{\circ} 39$, automne 2001, pp. 27-35.

52. Phrase relevée lors l'un échange verbal avec un groupe de Franco-Tunisiens à Paris en février 2011. 
la catégorie des "profanes", ces Franco-Tunisiens qui n'ont jamais fait de politique en France, véhiculant davantage une vision humanitariste de la relation à la société d'origine (II notre objectif n'est pas de faire de la politique, mais d'aider notre pays à s'en sortir $11^{53}$ ).

Ce type de débats passionnels sur la légitimité à se revendiquer tunisien a traversé la quasi-totalité des associations nées après le 14 janvier: " Oui, il y avait des différends assez profonds notamment avec les anciens étudiants du bled qui étaient arabophones. Nous, nous ne l'étions pas, et ils nous le faisaient comprendre. En somme, ils nous faisaient comprendre que, eux, ils étaient légitimes, et pas nous [...]. II y avait aussi des différends sur la question de l'islam. Nous, les Français, ils nous accusaient d'être islamophiles. Ils nous rétorquaient que, eux, ils connaissaient les islamistes sur le terrain et qu'ils voyaient leurs dégâts. En gros, les blédards nous accusaient d'être pro-islamistes. La question de l'islamisme a provoqué un conflit assez violent [...]. Par ailleurs, les mecs du bled avaient une vision élitiste des choses. Ils avaient tendance à avoir du mépris à l'égard des Tunisiens de base. Ils développaient un certain complexe de supériorité. Généralement, ils ne sont pas restés dans notre association. Ils sont partis $11^{54}$.

Les multiples conflits entre ces différentes catégories de militants de l'immigration expliquent sans doute le caractère profondément instable de l'espace associatif tunisien en France, dont la recomposition ne fait que commencer.

\section{Un espace politique imaginaire en réaction aux discri- minations ici ef là-bas ?}

Notre démarche visant à replacer les mobilisations protestataires des Franco-Tunisiens dans une perspective historique n'a pas simplement pour objectif de livrer aux lecteurs un récit héroïque des combats et des luttes politiques en immigration, mais surtout de comprendre les ressorts actuels de l'engovement des acteurs diasporiques à la fois pour la "Révolution" et pour le processus de transition démocratique, ces derniers s'inscrivant nécessairement dans une histoire longue qui ne saurait se limiter aux événements protestataires des derniers mois. Le militantisme des "nouvelles générations", s'il est porteur d'innovations pro-

53. Ibidem.

54. Wajdi Limam, président de l'UNIT. 
testataires, est aussi l'héritier de répertoires de mobilisation et de mémoires militantes enracinés dans l'expérience migratoire des individus et des familles.

Loin des interprétations binaires en termes d'allégeance/intégration ou des analyses psychologisantes sur le malaise identitaire des "nouvelles générations", nous avons voulu comprendre les motivations des héritiers de l'immigration à s'engager pour les causes sociopolitiques du pays d'origine. Or, du point de vue des acteurs diasporiques, les scènes politiques de la société du pays d'immigration et de la société du pays d'origine apparaissent comme totalement imbriquées, moins sur le plan structurel (la distinction entre la vie politique française et la vie politique de l'État d'origine est clairement établie par les acteurs) que sur le plan de l'imaginaire : les combats "ici" et "là-bas" ne se font plus sur des lignes de fracture supposant des choix irréductibles entre l'un et l'autre, mais souvent sur des problématiques communes : le refus des conceptions restrictives de la citoyenneté, des visions puristes de l'identité nationale ou des tendances larvées à réhabiliter le droit du sang dans la législation des États.

Sur ce plan, les acteurs diasporiques sont bien porteurs d'un rapport spécifique au politique qui se nourrit de l'expérience commune de la discrimination dans la société du pays d'immigration comme dans la société du pays d'origine, qui tend à les nier dans leur pleine citoyenneté $^{55}$ : en France, considérés comme des éternels immigrés, en Tunisie, comme des Ould el França. Mais loin de s'y résigner et d'être réduits à l'état d'impuissance permanent et d'entre-deux identitaire, ils entendent transformer leur binationalité en ressource de leur engagement à la fois individuel et collectif "ici" et "là-bas".

55. Manifeste des bi-nationaux tunisiens. Ne touchez pas à nos appartenances multiples !, 2011, http://www.fichier-pdf.fr/2011/12/17/le-manifeste-des-bi-nationaux-tunisiens 